\title{
Crisis de representación de los partidos politicos en el Perú
}

\section{Politic Parties Representation Crisis in the Peru}

Milton Ovidio Pastor Neyra*

http://dx.doi.org/10.21503/lex.v15i20.1452

* Lic. en Ciencia Política, lic. en Educación (especialidad de Filosofía y Ciencias Sociales), magíster en Ciencia Política, diplomado en Gestión de la Educación, estudios de doctorado en Ciencias de la Educación; analista político, conferencista y docente universitario.

Correo electrónico: milovipas@hotmail.com; m_pastor@doc.uap.edu.pe

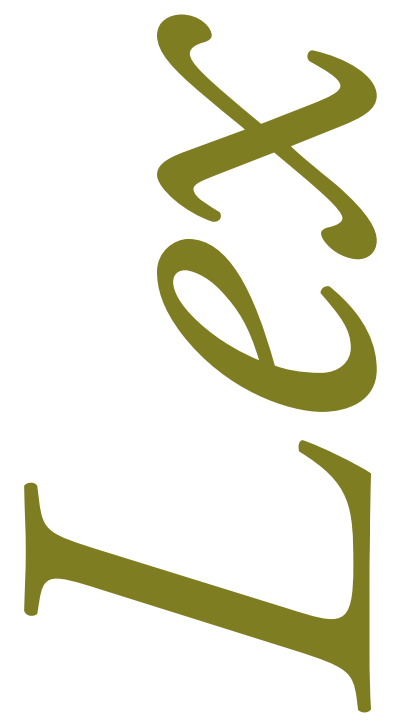




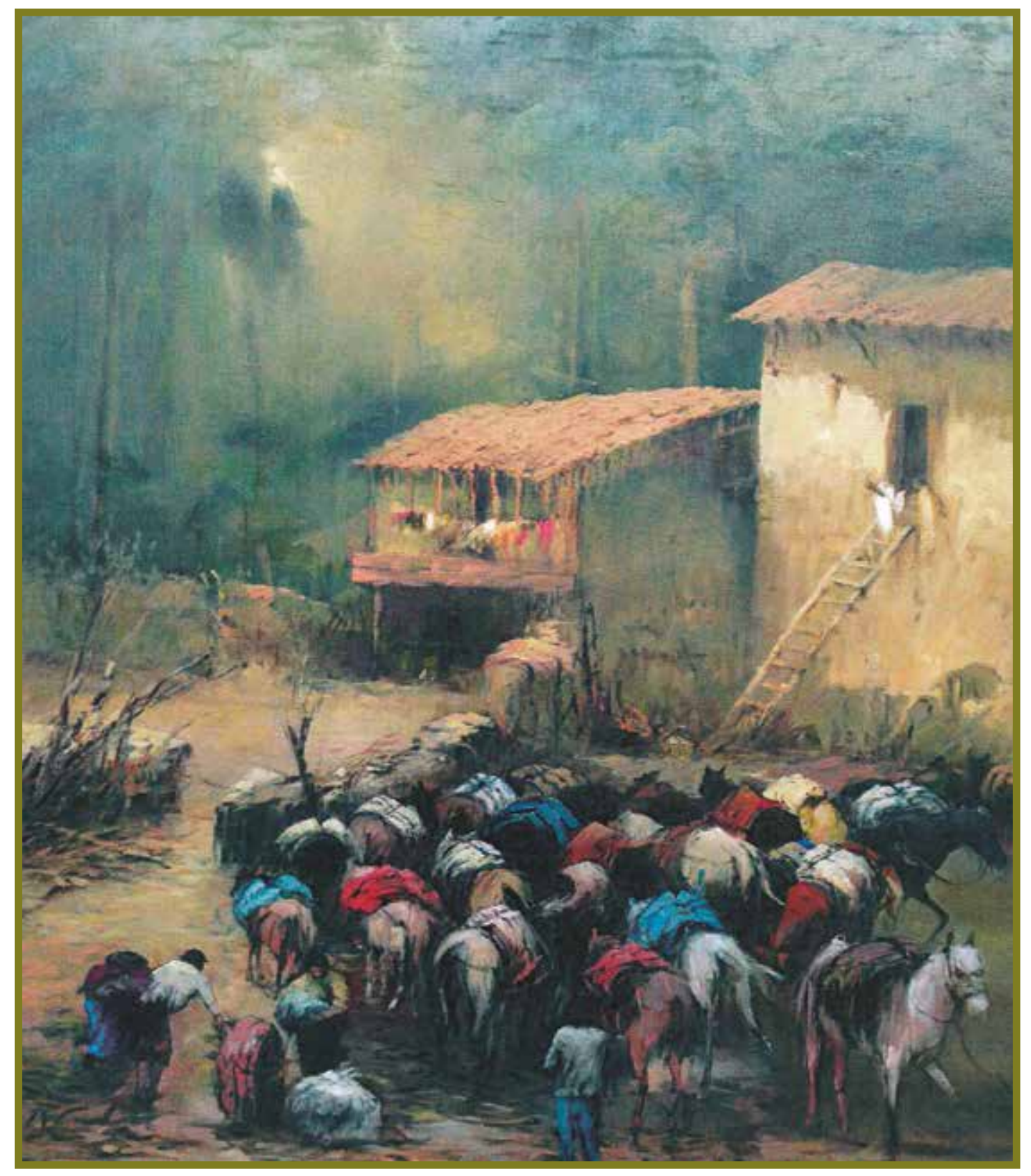

Raúl Cárdenas. La partida. 120 x 88 cm. 


\section{RESUMEN}

El presente artículo busca explicar la "crisis de representación de los partidos políticos en el Perú", sus causas y consecuencias, delimitando el escenario temporal a las décadas de los 80 y 90 . Trata de explicar cómo la crisis de los partidos político genera la aparición de los outsiders y a su vez explica cómo la crisis de los partidos políticos tiene como causa importante la crisis del linkage (enlace) entre la ciudadanía y el Estado. Contrasta aportes teóricos de diversos cientistas políticos, para explicar la aparición de los outsiders y la crisis de representación de los partidos políticos tradicionales en diferentes partes del mundo y en particular en América Latina y el Perú. Postula como causa principal de la crisis del linkage los cambios estructurales en la sociedad global, latinoamericana y peruana, que ha generado la emergencia de nuevos grupos sociales con nuevas demandas que no fueron advertidos por los actores institucionales del sistema político. En el caso peruano, esta crisis permitió la proliferación de los movimientos sociales y el surgimiento de los outsiders, que le dieron consistencia a la "antipolítica" como práctica política vigorosa en la década de los 90. También recomienda complementariamente, el liderazgo "transaccional" respecto al liderazgo "transformacional" para despersonalizar los partidos y fortalecer la institucionalidad del Partido y por ende del sistema político.

Palabras clave: outsiders, linkage, antipolítica, liderazgo transaccional, liderazgo transformacional.

\section{ABSTRACT}

This paper aims to explain the Political Parties' Representation Crisis in Peru, its causes and consequences, during the timeframe of the 1980 and 1990 decades. The author describes how the political parties' crisis led to the rise of outsiders and also explains how such crisis was mainly caused, among other causes, after the crisis on the linkage between the people and the Government. Contributions from different Political Scientists are contrasted in order to explain the appearance of the outsiders and the representation crisis of the traditional political parties in different countries around the world and particularly in Latin America. The main causes of the crisis of the linkage or connection between the people and the Government are: structural changes in the society on a Peruvian, Latin-American and Global scale, which generated the emergence of new social groups with new demands not previously noted by institutional actors from the political system. In the Peruvian case this crisis allowed the bloom of social movements and the rise of the outsiders strengthening the "Anti-Politics" as a strong political practice during the 1990 decade.

The author also recommends, complementing, the "transactional" leadership with respect to the "transformational" leadership in order to depersonalize the political parties and to strengthen the institutionalization of the Political Party and therefore of the political system.

Key words: outsiders, linkage, anti-political, transactional leadership, transformational leadership. 


\section{ANTECEDENTES}

Según Lipset y Rokkan, ${ }^{1}$ una vez que los sistemas de partidos son establecidos, las lealtades de los votantes se congelan. Dicha afirmación implicaría la poca probabilidad de que nuevos partidos políticos puedan surgir y consolidarse dentro de un sistema ya existente; sin embargo, tanto en el Perú como en otras partes del mundo durante los años 80 y 90 aparecieron figuras en la política que surgían de ámbitos distintos al de los partidos existentes, ya sea desde los mismos medios de comunicación o desde otros ámbitos sociales. El surgimiento de estas figuras generalmente además estuvo asociada a un discurso antipartido, llegándose a conocer en el caso peruano como independientes. Figuras políticas que surgieron a partir de estos nuevos partidos son los llamados outsiders, es decir, candidatos que no se encontraban considerados al inicio del proceso electoral como alternativas viables para ganar la elección, pero que terminaron en muchos casos ganándola o quedando en un lugar expectante al finalizar dicho proceso electoral.

Un examen de otros países revela que en varios casos nuevos partidos políticos han aparecido y se han consolidado. Si las lealtades de los votantes son tan estáticas, como sostenían Lipset y Rokkan, ¿por qué han aparecido estos nuevos partidos? Y ¿cuál ha sido la influencia que ha tenido la crisis de los partidos en la aparición de los outsiders? Estas son dos preguntas esenciales que se intenta abordar en el desarrollo de esta investigación.

Lipset y Rokkan no anticiparon la posibilidad de que los partidos políticos pudieran entrar en crisis, la cual abriría la puerta a nuevos competidores. Esta crisis, entonces, es un factor necesario, pero no suficiente, para la aparición y consolidación de nuevos partidos y outsiders en la política nacional.

1 Seymour Martin Lipset; Stein Rokkan, Party Systems and Voter Alignments: Cross-National Perspectives (New York: The Free Press, 1967). 


\section{LA CRISIS DE LOS PARTIDOS POLÍTICOS}

La propuesta de Lipset y Rokkan sobre sistemas de partidos y lealtades de los votantes parte de la idea de que necesariamente los partidos políticos son los principales vehículos para canalizar las demandas públicas y representar los múltiples intereses de la sociedad; sin embargo, en los últimos veinte años se ha visto cómo esa función ha sido asumida por otros entes sociales como los medios de comunicación o los frentes sociales, y aun cuando los partidos se convirtieron en partidos "atrapa-todo", que deberían ser partidos multiclasistas, con estructuras poco concretas y con un gran atractivo, como los denomina Kirchheimer. ${ }^{2}$ Pero su capacidad real para reflejar los intereses sociales cada vez ha ido reduciéndose, lo que ha permitido la aparición de nuevos fenómenos políticos en el escenario nacional.

Fueron pocos los politólogos que previeron que vendría el día en que esta función sería en gran parte abandonada por los partidos políticos. Los partidos políticos empezaron a entrar en crisis cuando llegaron a ser incapaces, o no tuvieron la voluntad, para cumplir sus funciones. Lawson, ${ }^{3}$ entre otros, reconoció esta tendencia. Ella sugirió que los partidos "fracasan" cuando dejan de ser linkage (enlace) entre la ciudadanía y el Estado, y ese fracaso se manifiesta generalmente en los resultados electorales con la victoria de figuras hasta ese momento poco conocidas en el ámbito partidario.

Lawson elabora más sobre el concepto, explicando que existen cuatro tipos de linkage separados, pero no mutuamente exclusivos, que utilizan los partidos políticos: participativo, electoral, clientelista y directivo. En el contexto latinoamericano, los dos tipos más comunes son el electoral y el clientelista. El primero exige que los candidatos respondan a las demandas constituyentes, pero movilicen a sus afiliados solamente para tareas electorales. El segundo consta del arreglo clásico de "votos por favores". Los dos tipos son vulnerables a la crisis; el linkage electoral fracasa cuando los oficiales elegidos no responden a las demandas constituyentes, mientras que el linkage clientelista falla cuando la red patrón-clientela se deshace, por una variedad de razones.

Lawson subraya que cuando los partidos fracasan en el sentido de linkage, surgen organizaciones alternativas antes del proceso electoral, o se habla de outsiders cuando este fenómeno se da dentro del mismo proceso electoral, aunque estas no necesariamente perduran. En su obra, Lawson sugirió que los nuevos partidos surgen después de una crisis sistémica, la cual podría

\footnotetext{
Según Kirchheimer, "al abandonar los intentos al encadrement intelectual y moral de las masas (el partido atrapa-todo) vuelve la atención cada vez más al escenario electoral, tratando de cambiar la efectividad a fondo por una audiencia más amplia y mayores éxitos electorales". Según Kirchheimer, "al abandonar los intentos al encadrement intelectual y moral de las masas (el partido atrapa-todo) vuelve la atención cada vez más al escenario electoral, tratando de cambiar la efectividad a fondo por una audiencia más amplia y mayores éxitos electorales". Peter Mair, ed. The West European Party System (Oxford: Oxford, University Press, 1990), 52.

3 Kay Lawson, The Comparative Study of Political Parties (New York: St. Martin's Press, 1976).
} 
ser resultado de problemas como la legitimidad, la integración o la participación. En otras palabras, los nuevos partidos pueden aparecer después de un cambio de régimen (legitimidad), adquisiciones territoriales (integración) o demandas sociales crecientes (participación). En el contexto latinoamericano, son más comunes las primeras y terceras crisis, ya que la mayoría de estas naciones no han experimentado serios ajustes territoriales desde hace casi un siglo.

La crisis de los partidos no solo puede ser sistémica sino endémica, es decir, gradual, y puede ser producida por varios factores dentro de los cuales se encuentra la modernización. Los procesos continuos de desarrollo social y, cómo no, la falta de una voluntad política real por parte de las élites para mejorar las condiciones de participación de la ciudadanía en el proceso de toma de decisiones políticas.

América Latina, y el Perú de manera particular, hoy en día es urbana, educada, industrializada y pasa por profundos cambios estructurales. Como resultado, están emergiendo nuevas demandas dentro de la sociedad, que no encuentran, necesariamente, representación en los partidos tradicionales, muchos de los cuales siguen utilizando la práctica obsoleta del clientelismo. Aún más, la sociedad moderna es sustantivamente más atomizada que antes, y el achicamiento del Estado ha contribuido a lo que llama Rial "el analfabetismo político", lo cual, a su vez, ha creado un tipo de desconfianza en la clase política en general, pero en particular en aquellas personas percibidas como vinculadas al viejo orden. Rial y otros autores ven una correlación entre el desprestigio creciente de los partidos tradicionales y la aparición del fenómeno outsider, encarnado por líderes como Alberto Fujimori en los noventa y Ollanta Humala en el nuevo milenio.

En Exit, Voice and Loyalty, la obra corta pero bien reconocida de Hirschman, ${ }^{5}$ se dice que los ciudadanos inconformes, como los consumidores, son leales a su partido, solo con tal de que se sientan satisfechos con su desempeño. Cuando dejan de estar satisfechos pueden expresar su malestar con la intención de reformar el partido, pueden salir del partido, buscar otro partido que mejor represente sus ideas, o simplemente estar apáticos cuando no encuentran alternativa. Este comportamiento nos ayuda a explicar la crisis actual de los partidos políticos. Muchos votantes en América Latina y en otros lugares del mundo han decidido dejar su partido, después de haber llegado a estar insatisfechos con el desempeño de este, aunque, claro, en el caso peruano la presencia de una alta volatilidad electoral es una muestra de la falta de identificación partidaria que casi desde siempre han arrastrado los partidos y que hace aún más propicio el escenario para la aparición de outsiders en las contiendas electorales.

4 Juan Rial, "Los partidos políticos en América del Sur en la primera mitad de los años noventa”, en Partidos y clase política en América Latina en los 90, compilado por Carina Perelli, Sonia Picado S. y Daniel Zovatto (San José: Instituto Interamericano de Derechos Humanos, Centro de Asesoría y Promoción Electoral [Capel]), 1995.

5 Citado por Lawrence Boudon, "Hacia una teoría sobre nuevos partidos políticos”, en Sección Temas Globales: 2. 
Parece, entonces, que Lipset y Rokkan se equivocaron. Una vez que se forman los sistemas de partidos, las lealtades de los votantes no llegan a congelarse. Las crisis, sean sistémicas o endémicas, tienden a perturbar las cosas que en algún momento parecían estar estables. Los partidos establecidos, de cualquier tipo, muchas veces son incapaces de superar las crisis, bien porque la crisis es demasiado severa, o porque no están bien dotados para adaptarse a las circunstancias cambiantes. Los partidos de masas pueden estar demasiado rígidos estructuralmente para permitir un ajuste fácil, mientras que los partidos "atrapa-todo" tal vez no poseen una organización suficiente para mantener el linkage. Así es que Rose y Mackie, en su estudio multinacional de partidos políticos, ${ }^{6}$ descubrieron que los partidos antiguos tenían menos posibilidades para perdurar que los partidos que aparecieron después de una crisis.

Una crisis de partidos o sistemas, entonces, parece ser la condición necesaria para la aparición de nuevos partidos políticos y el surgimiento de outsiders, pero, como dice Lawson, no basta para que persistan. Su estudio reveló que organizaciones alternativas (incluyendo partidos políticos) no perduran solamente por proveer el mismo tipo de linkage abandonado por los partidos tradicionales, aunque sí encontró que estas organizaciones adoptan el tipo de forma que les permitiría hacerlo. Curiosamente, Lawson descubrió que es más probable que estas organizaciones provean este linkage, a que desaparezcan. La razón parece ser que los partidos tradicionales adoptaron muchas de las políticas y prácticas asumidas por estas organizaciones, utilizando así un tipo de cooptación. Por lo tanto, el comportamiento de los partidos existentes es una variable importante para considerar, ya que los nuevos partidos no aparecen de la nada.

En suma, aunque los fracasos de linkage de los grandes partidos pueden resultar en la creación de organizaciones alternativas que son bien diseñadas para llenar el vacío de linkage así producido, la capacidad de estas organizaciones para prosperar y perdurar parece depender tanto de las variables externas - la naturaleza de otros partidos, otras instituciones y actitudes populares - como de factores dentro de su propio control.

\section{PERSISTENCIA Y FRACASO}

Una variable obvia que se debe considerar con respecto a nuevos partidos políticos es el liderazgo. El tipo de liderazgo que posee un partido puede ser vital para su éxito, ya que son los líderes quienes están más visibles frente al público votante. Duff postula que un liderazgo "transaccional" es preferible al "transformacional", en términos de construir un partido cohesivo. En otras palabras, los políticos con capacidad para negociar y construir

\footnotetext{
Citado por Lawrence Boudon, "Hacia una teoría sobre nuevos partidos políticos”, en Sección Temas Globales: 4.

Ernest Duff, Leader and Party in Latin America (Boulder: Westview Press, 1985).
} 
una organización benefician el partido más a largo plazo que los líderes carismáticos, cuyos talentos se basan en hacer apelaciones emocionales a las masas. La tesis de Duff es que los líderes transformacionales tienden a crear un culto de personalidad que los hace indispensables para el partido. Es decir, construyen partidos personalistas que casi nunca sobreviven después de su fallecimiento. Los líderes transaccionales, en cambio, dejan una organización de larga duración.

Cuando un nuevo partido entra en un sistema existente, por ejemplo, es más importante desde el principio poder distinguir ese partido de los demás, una tarea mejor llevada a cabo por un líder carismático. Por otro lado, si el líder se ve negociando con los partidos existentes y desprestigiados, habrá menos posibilidad de que los votantes descontentos salgan y escojan ese nuevo partido. De otra parte, los líderes transaccionales quedan más vulnerables a la cooptación, ya que se enfocan más en la posibilidad de entrar en una coalición que en formular una ideología concreta y coherente.

Es totalmente posible que un nuevo partido esté agraciado con un líder que es a la vez carismático y pragmático, pero Rose y Mackie argumentan que los líderes de partidos enfrentan un gran trade-off entre cuánto tiempo y esfuerzo pueden dedicar a las presiones internas y externas. En otras palabras, los líderes de partidos deben intentar simultáneamente construir una organización cohesiva (interna), mientras tratan de conseguir votos (externa). Rose y Mackie consideran que este trade-off es un juego de suma cero; los líderes tienen que cambiar el uno por el otro, no pueden hacer los dos a la vez.

En suma, entonces, un liderazgo carismático es preferible inicialmente, ya que no solamente permite al partido distinguirse de los ya existentes, sino que enfatiza la organización interna, uno de los tres requisitos para la institucionalización.

Hauss y Rayside ${ }^{8}$ han sugerido también otras tres variables, las cuales, creen ellos, han influido en el éxito y fracaso de los nuevos partidos políticos: 1) el comportamiento de los partidos existentes, 2) el compromiso de las masas con un cambio político, y 3) la organización de los nuevos partidos. De estas, ellos argumentan que la primera puede ser la más importante, ya que los partidos existentes poseen varios mecanismos que pueden usar para apretar a los nuevos partidos. Aquellos pueden reformar las reglas electorales en su propio beneficio, pueden negar el reconocimiento legal, o pueden simplemente empeñarse en la cooptación, la cual puede encarnarse en una de dos formas. Primero, los partidos existentes pueden, en esencia, usurpar la posición programática ocupada por el nuevo partido, es decir, robar sus ideas y presentarlas como propias. Budge, Robertson y Hearl ${ }^{9}$ sugieren en

8 Charles Hauss y David Rayside, “The Development of New Parties in Western Democracies Since 1945”, en Political Parties: Development and Decay, editado por Louis Maisel y Joseph Cooper (Beverly Hills: Sage Publications, 1978$), 37$.

9 Ian Budge and David Robertson, "Do parties differ, and how? Comparative discriminant and factor analysis 1987", in 
su análisis espacial multinacional que se puede calificar este tipo de comportamiento como cooptación. Segundo, los partidos existentes pueden tratar de cortejar el liderazgo del nuevo partido ofreciendo cargos gubernamentales importantes. Tercero, la cooptación puede manifestarse a través de las coaliciones. Puede ser que un nuevo partido con orientación radical, por ejemplo, tenga que moderarse una vez que se desempeña en el acto de gobernar. Esta forma de cooptación tiene además la ventaja de convertir a los outsiders en insiders, y así hacerlos parte de la crisis.

La segunda variable mencionada por Hauss y Rayside —el compromiso de las masas con el cambio político - también es fundamental para la permanencia de los nuevos partidos, ya que sin un electorado que ha escogido la opción de abandonar, los nuevos partidos tienen pocas posibilidades. Aunque es difícil medir los sentimientos de los votantes fuera de las encuestas, se puede decir que esta variable es meramente una reiteración de nuestra condición necesaria, pero no suficiente: una crisis sistémica o partidaria. Las señas de apatía electoral o el descontento abierto con los partidos existentes, combinado con las crisis persistentes políticas o económicas, pueden servir como evidencia de este compromiso con el cambio político. También lo puede ser la voluntad de los votantes para apoyar a los outsiders.

Seguramente hay otros factores que influyen en el éxito y fracaso de los nuevos partidos. El listado no se agota aquí. Unas variables son aquellas que se podrían llamar externas o circunstanciales. Entre estas existe el tipo de sistema electoral, la disponibilidad de modelos en el sistema partidario actual, unas crisis políticas y/o económicas persistentes y los constreñimientos ideológicos.

No hay mucho desacuerdo entre los académicos sobre el impacto de las reglas electorales en cuanto al éxito de los nuevos partidos. Como se dijo al principio, una de las razones por las cuales estos han recibido tan poca atención en los Estados Unidos y en Gran Bretaña es que el esquema de la uninominalidad y la mayoría absoluta impiden severamente su institucionalización. Los dos países usan la uninominalidad, bajo la cual el candidato que recibe más votos que los demás gana el escaño. El efecto de esto en los votantes es sencillo. Aunque ellos tal vez prefieren un tercer candidato, votarán por uno de los dos candidatos pertenecientes a los partidos tradicionales, por temor de desperdiciar su voto, creyendo que su candidato no tiene ninguna posibilidad de ganar.

Es evidente, entonces, que la representación proporcional tendería a favorecer, o por lo menos a no perjudicar a los nuevos partidos políticos y favorecería la aparición del outsider.

Ideology, strategy and Party change: spatial analysis of post-war election programmes in 19 democracies, ed. by Ian Budge, David Robertson y Derek Hearl (Great Britain: Departament of Government, University of Essex and St Hugh's College, University of Oxford 1987), 387. 
Cuando aparecen los nuevos partidos, como se ha dicho antes, no entran al vacío. Existen ya otros partidos que sirven como modelos para los nuevos. Por eso, el tipo de modelo disponible en el sistema puede ser una variable importante, según Ware, ${ }^{10}$ quien arguye que los nuevos partidos tienden a copiar lo que ven; claro, eso no significa que copien necesariamente a los partidos tradicionales, sino a aquellos que han tenido éxito electoral; de tal forma podemos ver cómo luego del éxito electoral del movimiento independiente "OBRAS" de Ricardo Belmont Cassinelli en los subsiguientes procesos electorales se fueron incrementando los movimientos independientes y con ello la aparición de nuevas figuras en la arena política, aun cuando muchas de esas figuras ya habían pertenecido a organizaciones políticas de corte tradicional. En ese sentido, entonces, si un nuevo partido entra en un sistema que contiene solamente partidos clientelistas con poca organización, es probable que este también llegue a ser clientelista, con poca organización. La lógica es: "si funciona para ellos, debe funcionar también para nosotros". Desafortunadamente, al imitar a los partidos existentes, el nuevo, sin saberlo, puede seguir el mismo camino que lo condujo a la crisis en el principio; los partidos tradicionales no mantuvieron su linkage.

Para persistir, entonces, los nuevos partidos deben evitar la tendencia a copiar los modelos existentes, siempre y cuando haya sido el modelo en sí la causa del problema. Por otro lado, si los partidos existentes están en crisis, debido a problemas sistémicos, como un escándalo político, el imitarlos tal vez no sería tan perjudicial, en particular si se puede caracterizar a estos partidos como bien organizados y con bases locales de apoyo. La tarea aquí es poder copiar la estructura del partido, sin imitar el comportamiento que condujo a la crisis, la cual es otra variable.

En América Latina, encontramos que el avance de distintos movimientos y de candidatos antipolíticos se dio tanto en aquellos países que contaban con partidos y sistemas de partidos estables y organizados como Colombia y Venezuela, como en aquellos países con partidos y sistemas de partidos fragmentados, débiles y desorganizados como Perú, Bolivia, Ecuador y Brasil. La antipolítica, como nueva forma de hacer política en los noventa estuvo a la ofensiva, aprovechando la situación de cuestionamiento de los actores, expresándose, entre otras cosas, como "una reestructuración de los universos y prácticas políticas en el sentido en que se redefine la relación entre los ciudadanos y la política”.

De tal modo que la antipolítica, como lo ha destacado René Antonio Mayorga en su estudio comparativo de Bolivia, Brasil y Perú, se desarrolla paradójicamente como una forma de hacer política que pretende no solo prescindir de los partidos políticos, sino también pone en cuestión las pautas predominantes del quehacer político de los Gobiernos democráticos.

10 Alan Ware, Citizens, Parties and the State: A Reappraisal (Princeton: Princeton University Press, 1987). 
Asimismo, no debemos perder de vista que en países tales como Bolivia, Perú, Brasil y Venezuela el surgimiento de candidatos outsiders o extra-partido y el impacto de los mismos en la nueva política democrática latinoamericana ha coincidido con una situación de crisis económica, de ingobernabilidad y de cuestionamiento de las élites políticas. El avance de la llamada antipolítica como nueva política coincide con una suerte de "fatiga cívica" del propio sistema. En tal sentido, esta situación ha provocado el surgimiento de outsiders en casi toda la región, dentro de un espectro político caracterizado por la confusión, el descrédito de los partidos políticos y el agotamiento de las instituciones democráticas.

El avance de la antipolítica revela también la transformación de la política y la presencia de una nueva cultura política, en la que la política como instancia privilegiada de representación y coordinación de la vida social se ha vuelto problemática, y como instancia central de lo social tiende a desvanecerse. Por consiguiente, creemos que este fenómeno exige un replanteamiento de lo público y lo privado, y, por sobre todo, demanda reformulación en la tarea de repensar la política, a fin de retomar y reexaminar sus contenidos.

\section{LOS OUTSIDERS COMO ALTERNATIVA A LA CRISIS DE REPRESENTACIÓN}

Como dirían Kay Lawson y Peter Merkl, ${ }^{11}$ las alternativas a los partidos políticos aparecen cuando el vínculo o intermediación establecida por ellos falla en establecer una relación aceptable entre los ciudadanos y el poder. Cuando decimos aceptable nos estamos refiriendo a un tipo de relación que satisfaga las necesidades de legitimación que los individuos asumen que una determinada forma de poder político debe tener.

Estas alternativas a la crisis de representación, vía los partidos políticos, en las diferentes partes del mundo han sido principalmente dos: los denominados movimientos sociales y el surgimiento de independientes u outsiders. No podemos decir que unos u otros hayan sido exclusivos de tal o cual parte del mundo. Tenemos de ambos tanto en las democracias establecidas como en las que están en proceso de consolidación. Silvio Berlusconi en Italia, Ross Perot en los Estados Unidos, Alberto Fujimori en el Perú, Irene Sáenz en Venezuela, Palito Ortega en la Argentina; los movimientos por los derechos humanos en la América Latina que iniciaron las transiciones a la democracia en los setenta y los ochenta, los movimientos ecologistas, feministas y pacifistas en los Estados Unidos y Europa Occidental, los foros cívicos contra el totalitarismo comunista en Europa Oriental, etc. Además, en cada caso hay diferentes variedades y distinto grado de éxito político.

11 Kay Lawson y Peter Merkl, eds., When Parties Fail: Emerging Alternatwe Organizations (Princeton: Princeton University Press, 1988). 
Detrás de cada una de estas alternativas hay diagnósticos en alguna medida también diferentes. Quienes promueven los movimientos sociales suelen apuntar al carácter elitista de las democracias modernas, o estar enfrentando regímenes autoritarios. La alternativa, en este caso, es una relación más directa con el poder vía la participación de los ciudadanos en la toma de las decisiones que afectan a la población. Quienes resaltan la irrupción de los outsiders, por otra parte, suelen considerar a los partidos en sí mismos como la fuente de los problemas de la democracia, resaltando la necesidad de la población de una comunicación directa con quien toma las decisiones políticas. Tenemos entonces que, como ilusión o realidad, lo que se buscaría es una relación más cercana de la ciudadanía con el poder.

Si tomamos el caso de los movimientos sociales, es decir, de la acción colectiva y organizada de un conjunto de personas con un propósito específico, vemos que adquieren relevancia política porque los partidos no han podido, querido o sabido levantar esa reivindicación o conjunto de reivindicaciones y plantear una solución al respecto. En este sentido, los movimientos sociales ganan vigencia política en forma rápida y puntual, dejando de lado a los partidos. Es más, estos movimientos suelen ganar simpatía más allá de sus miembros porque tienden a fortalecer el tejido social, dando consistencia a lo que se ha venido en llamar la sociedad civil, o el sector organizado de la sociedad, base de la interacción política en las democracias representativas. A veces se tiende a confundir movimiento social con grupo de interés, pero si bien el primero está incluido en el segundo, la característica que distingue a los movimientos sociales es la importancia de la movilización colectiva de sus miembros para conseguir sus objetivos. Por ello, generalmente se usa la denominación movimiento social para referirse a los movimientos surgidos de la sociedad civil que luchan por una agenda específica de demandas y concitan la participación directa de los que conforman el movimiento.

Pero la alternativa que más nos interesa, por la importancia que ha tenido en el Perú en los últimos años, es la de los denominados independientes u outsiders.

\section{REFERENCIAS}

- Budge, Ian and David Robertson. "Do parties differ, and how? Comparative discriminant and factor analysis 1987". In Ideology, strategy and Party change: spatial analysis of post-war election programmes in 19 democracies, ed. by Ian Budge, David Robertson y Derek Hearl (Great Britain: Departament of Government, University of Essex and St Hugh's College, University of Oxford 1987).

- Duff, Ernest. Leader and Party in Latin America. Boulder: Westview Press, 1985. 
- Hauss, Charles y David Rayside. “The Development of New Parties in Western Democracies Since 1945". En Political Parties: Development and Decay, editado por Louis Maisel y Joseph Cooper. Beverly Hills: Sage Publications, 1978.

- Lawrence Boudon. "Hacia una teoría sobre nuevos partidos políticos". En Sección Temas Globales.

- Lawson, Kay. The Comparative Study of Political Parties. New York: St. Martin's Press, 1976.

- Lawson, Kay y Peter Merkl, eds. When Parties Fail: Emerging Alternatwe Organizations. Princeton: Princeton University Press, 1988.

- Lipset, Seymour Martin; Stein Rokkan. Party Systems and Voter Alignments: Cross-National Perspectives. New York: The Free Press, 1967.

- Mair, Peter, ed. The West European Party System. Oxford: Oxford, University Press, 1990.

- Rial, Juan. "Los partidos políticos en América del Sur en la primera mitad de los años noventa". En Partidos y clase política en América Latina en los 90, compilado por

- Carina Perelli, Sonia Picado S. y Daniel Zovatto. San José: Instituto Interamericano de Derechos Humanos, Centro de Asesoría y Promoción Electoral (Capel), 1995.

- Ware, Alan. Citizens, Parties and the State: A Reappraisal. Princeton: Princeton University Press, 1987.

Recibido: 05/06/2017

Aprobado: 07/10/2017 


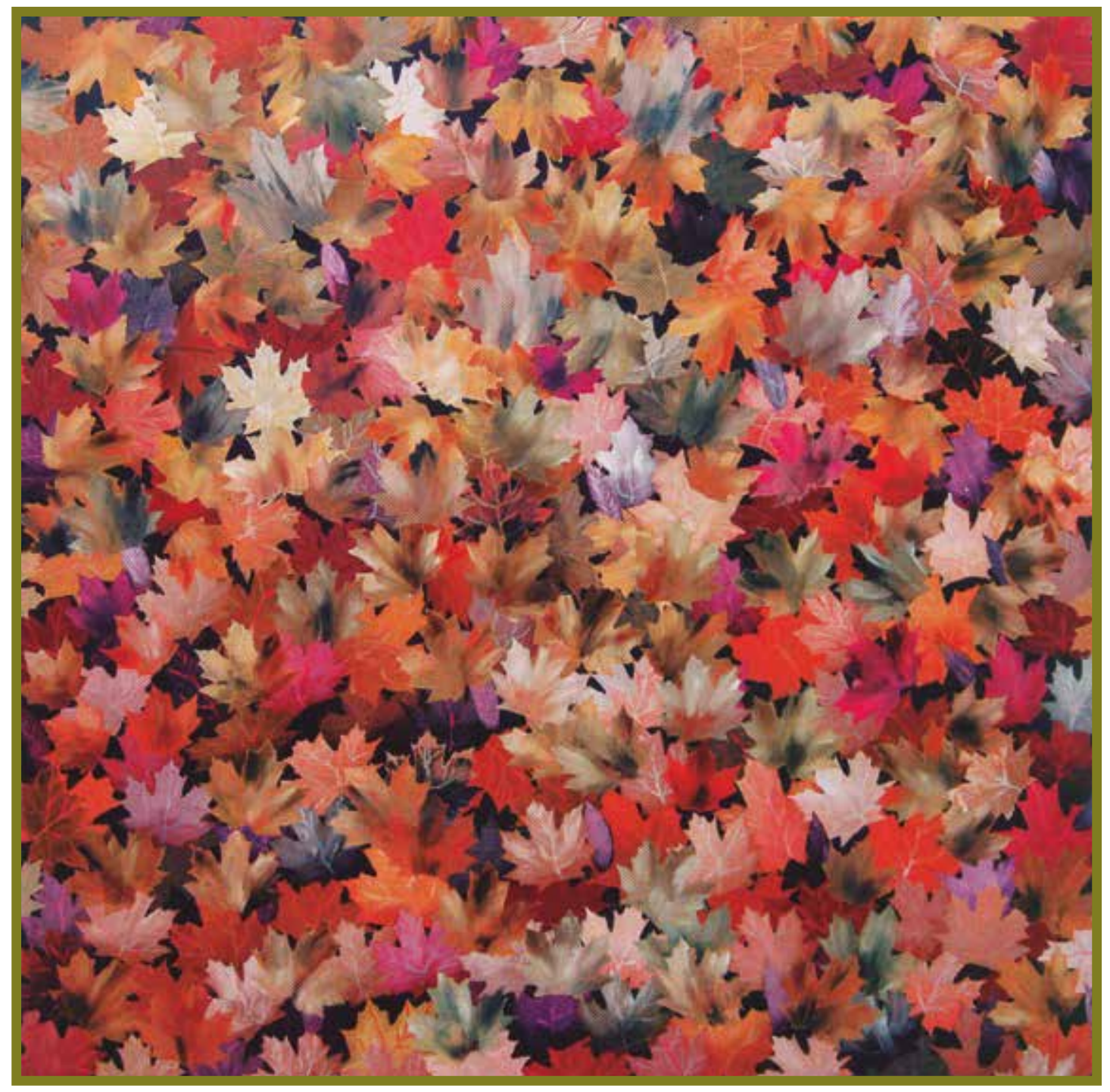

Raúl Cárdenas. Maple. 150 x $150 \mathrm{~cm}$. 